\title{
Ear and Face Mucormycosis; A Case Report
}

Khosravi $\mathrm{MH}^{1}$

Zahra $\mathrm{K}^{2}$

Hosseini $M^{3}$

Dadgar $\mathrm{S}^{4}$

Ziya $D^{5}$

Saeedi $\mathrm{M}^{6}$

\section{ABSTRACT}

Mucormycosis is an invasive fungal infection belonging to order of Mucorales which causes a high rate of mortality. This infection is mostly common in the immunosuppression conditions such as diabetes mellitus, chemotherapy, organ transplantation and hematologic malignancies.

Keywords: Mucormycosis, otolaryngology, swelling, facial skin.

${ }^{1}$ Department of Otorhinolaryngology Research Association (IORA), Universal Scientific Education and Research Network, Iran

${ }^{2}$ Student Research Committee, Semnan University of Medical Sciences, Semnan, Iran

${ }^{3}$ Student Research Committee, Qazvin University of Medical Sciences, Qazvin, Iran

${ }^{4}$ Medical Student, Faculty of Medicine, Tehran University of Medical Sciences, Tehran, Iran

${ }^{5}$ Department of Plastic and Reconstructive Surgery, Imam Khomeini Hospital, Tehran University of Medical Sciences, Tehran, Iran

${ }^{6}$ Department of Otorhinolaryngology-Head and Neck Surgery, Faculty of Medicine, Baqiyatallah University of Medical Sciences, Tehran, Iran

*Send correspondence to:

Masoumeh Saeedi,

Department of Otorhinolaryngology-Head and Neck Surgery, Faculty of Medicine, Baqiyatallah University of Medical Sciences, Tehran, Irana, E-mail: m.saeedi67@ gmail.com, Phone: +989122192388

Paper submitted on August 08, 2019; and Accepted on December 08, 2020 


\section{INTRODUCTION}

Histological examination of Mucormycosis reveals a necrotizing vasculitis which results in tissue infarction. The infection is transmitted to human most usually by inhalation of sporangiospores and sometimes through contaminated food or trauma ${ }^{1-3}$. A recent study reports that Mucormycosis is more prevalent in Europe (34\%) followed by Asia (31\%), America (28\%), Africa (3\%) and Australia and New Zealand (3\%) ${ }^{4}$. Despite medical interventions, prognosis of Mucormycosis is really poor with a reported mortality rate between $32 \%$ and $70 \%$; however, rapid diagnosis and accurate localization is accompanied with survival. We hereby report a female case of ear and face Mucormycosis who was diagnosed and survived by surgical and medical interventions.

\section{CASE PRESENTATION}

We describe a 66-year old female who attended to otolaryngology clinic of our hospital with chief compliant of pain and swelling of right side of the face. The pain had been started in right ear two days before attendance to clinic and following bath. Then pain and swelling were spread to right side of the face and she had noticed a change in the colour of facial skin the night before visit. The patient was a known case of poorly controlled Diabetes Mellitus (DM) and hypertension. Also she had a history of hospitalization with diagnosis of Guillain-Barré syndrome 14 years prior to this visit. Insulin, Metformin and Losartan were among the drugs used by the patient. Family and psychosocial history revealed no special information. In physical examination she was ill and a significant swelling and ecchymosis was seen in right auricle associated with bloody and plasmatic secretions from ear canal. The patient had a tense swelling in right parotid region which was extended to right mandibular angle. The left eye was blind and swelling of right infraorbital area was evident which was accompanied by painful eye movements. She had a poor oral hygiene and trismus was detected in evaluations. Neurologic examinations revealed peripheral paralysis of right facial nerve. After physical examination the patient was admitted for further evaluations. Axial brain computed tomography (CT) scan and magnetic resonance imaging (MRI) were requested for the patient. CT scan showed soft tissue density in right external and middle ear as well as under-pneumatization of mastoid cells in right side. MRI revealed increased signal and enhancement of right petromastoid sinus and middle ear. A heterogeneous enhanced mass was seen at right coronoid process, parapharynx and adjacent pterygoid. In addition, right parotid gland and external auditory canal showed hyposignal mass suggestive for malignant or infective process. Based on aforementioned findings and history of DM, the patient was transferred to operation room with impression of Mucormycosis the day after first visit. After inducing general anesthesia, an incision was made anterior to the right tragus and according to necrosis of skin, subcutaneous tissue and its extension to Parotid, the patient underwent right total Parotidectomy.
Then partial resection of soft tissue as well as Pterygoid, Temporalis and Maseter muscles was done to the level of Zygomatic bone for reaching a non-necrotic margin. In addition, right auricle was amputated due to necrosis. No primary repair was done and surgery site was dressed with sterile gauze. Patient was transferred to recovery room with stable vital signs. All the specimens as well as parotid and right auricle were sent for pathology assessment which was compatible with Mucormycosis. Patient was admitted in ICU after surgery and underwent treatment with intravenous antibiotics and antifungal agents. Meropenem (500 mg BID), Clindamycin (900mg TDS), Caspofungine (Daily) and liposomal Amphotericine (150mg Daily) were prescribed for the patient. The surgical wound underwent daily washing with Hydrogen Peroxide and Acetic Acid and necrotic tissues were debrided daily. The patient was discharged 10 days after surgery. The 6-month follow up was eventless.

\section{DISCUSSION}

Recent reports show that the incidence of Mucormycosis is increasing globally especially in patients with uncontrolled diabetes mellitus ${ }^{5}$. Mucormycosis may involve different anatomic sites including nose and sinuses, lungs, gastrointestinal tract, skin, kidney as well as other organs such as ear, parotid gland, heart, bones and urinary bladder. Rhino-Orbito-Cerebral Mucormycosis (ROCM) is the most prevalent form of the disease which mostly occurs in context of diabetic ketoacidosis or uncontrolled diabetes mellitus. Rhino-Orbito-Cerebral Mucormycosis has a wide range of symptoms from headache, fever and facial swelling to toothache, facial palsy and altered mental status ${ }^{6}$. Infection can spread to brain through sphenoid and ethmoid sinuses as well as cribriform palate or perineural route ${ }^{7}$. Rapid diagnosis in addition to emergent fungal and surgical therapy is the main action for increasing survival. A variety of imaging modalities can be used for determining the extent of invasion. Mucormysosis has various manifestations in Magnetic Resonance Imaging and Computed Tomography including bone destructions, sinusitis and mucosal thickening as well as osseous erosions. Cerebral and orbital extension of the infection can be seen as optic neuritis, orbital cellulitis, erosion of the skull-base, cavernous sinus thrombosis and brain abscess ${ }^{8}$. Mucormycosis is a life-threatening infection which involves almost always patients with immunosuppressive conditions. Risk factors of infection differ from a region to another; hematological malignancies are the most common risk factor in Europe and North America while uncontrolled diabetes mellitus is the main risk factor in developing countries 9,10. Rhino-orbito-cerebral and pulmonary Mucormycosis are the most prevalent forms of infection and the variety of clinical manifestations makes a delay in diagnosis. Appropriate and rapid diagnosis of Mucormycosis involves a comprehensive approach using imaging modalities, pharmacological therapy and surgery which may end with better prognosis and survival of patients. 


\section{CONFLICT OF INTEREST}

Author declares No conflict of Interest

\section{REFERENCES}

1. Serris A, Danion F, Lanternier $F$. Disease Entities in Mucormycosis. Journal of Fungi. 2019;5(1):23-4.

2. Liu M, Spellberg B, Phan QT, Fu Y, Fu Y, Lee AS, et al. The endothelial cell receptor GRP78 is required for mucormycosis pathogenesis in diabetic mice. The Journal of clinical investigation. 2010;120(6):1914-24.

3. Richardson M. The ecology of the Zygomycetes and its impact on environmental exposure. Clinical Microbiology and Infection. 2009;15:2-9.

4. Jeong W, Keighley C, Wolfe R, Lee WL, Slavin MA, Kong DC, et al. The epidemiology and clinical manifestations of mucormycosis: a systematic review and meta-analysis of case reports. Clinical Microbiology and Infection. 2018.

5. Prakash H, Chakrabarti A. Global epidemiology of mucormycosis. Journal of Fungi. 2019;5(1):26.

6. Vaughan C, Bartolo A, Vallabh N, Leong SC. A meta-analysis of survival factors in rhino-orbital-cerebral mucormycosishas anything changed in the past 20 years? Clinical Otolaryngology. 2018;43(6):1454-64.

7. Wali U, Balkhair A, Al-Mujaini A. Cerebro-rhino orbital mucormycosis: an update. Journal of infection and public health. 2012;5(2):116-26.

8. Therakathu J, Prabhu S, Irodi A, Sudhakar SV, Yadav VK, Rupa V. Imaging features of rhinocerebral mucormycosis: A study of 43 patients. The Egyptian Journal of Radiology and Nuclear Medicine. 2018;49(2):447-52.

9. Corzo-León DE, Chora-Hernández LD, Rodríguez-Zulueta AP, Walsh TJ. Diabetes mellitus as the major risk factor for mucormycosis in Mexico: Epidemiology, diagnosis, and outcomes of reported cases. Medical mycology. 2017;56(1):29-43.

10. Skiada A, Pagano L, Groll A, Zimmerli S, Dupont B, Lagrou $\mathrm{K}$, et al. Zygomycosis in Europe: analysis of 230 cases accrued by the registry of the European Confederation of Medical Mycology (ECMM) Working Group on Zygomycosis between 2005 and 2007. Clinical Microbiology and Infection. 2011;17(12):1859-67. 